\title{
Pathways involved in testicular germ cell apoptosis in immature rats after FSH suppression
}

\author{
Saleela M Ruwanpura ${ }^{1,2}$, Robert I McLachlan ${ }^{1,2}$, Peter G Stanton ${ }^{1}$, Kate L Loveland ${ }^{3}$ \\ and Sarah J Meachem ${ }^{1}$ \\ ${ }^{1}$ Prince Henry's Institute of Medical Research, Clayton, Victoria 3168, Australia \\ ${ }^{2}$ Department of Obstetrics and Gynaecology, Monash University, Clayton, Victoria 3168, Australia \\ ${ }^{3}$ Monash Institute of Medical Research and ARC Centre of Excellence in Biotechnology and Development, Clayton, Victoria 3168, Australia \\ (Correspondence should be addressed to S J Meachem; Email: sarah.meachem@princehenrys.org)
}

\begin{abstract}
$\mathrm{FSH}$ is a key regulator of testis function, required for the establishment of full complements of Sertoli and germ cells during postnatal testis development and for the maintenance of spermatogenesis in the adult. FSH plays an important role in germ cell survival rather than proliferation, in the window between 14 and 18 days of testicular development, which coincides with the cessation of Sertoli cell proliferation and the onset of germ cell meiosis during the first wave of spermatogenesis. This study aimed to identify the pathway(s) of apoptosis regulated by changes in FSH levels in 14- to 18-day-old rats, using a model of in vivo FSH suppression by passive immunoneutralization with a rat anti-FSH antibody. Apoptotic pathways were identified by immunohistochemistry using pathway-specific proteins as markers of the intrinsic (activated caspase 9) and extrinsic (activated caspase 8) pathways, followed by quantification of cell numbers using stereological techniques. In addition, RT-PCR was used to
\end{abstract}

assess the expression of pathway-specific genes. We previously reported a $2 \cdot 5$-fold increase in spermatogonial apoptosis in these samples after 4 days of FSH suppression, and now show that this increase correlates with a $9 \cdot 8$-fold $(P<0 \cdot 001)$ increase in the frequency of caspase 9-positive spermatogonia in the absence of caspase 8 immunoreactivity. By contrast, spermatocytes exhibited both increased caspase 9 (7.5-fold; $P<0 \cdot 001)$ and caspase $8(5 \cdot 7$ fold; $P<0 \cdot 001)$ immunoreactivities after 4 days of FSH suppression. No significant change in the transcription levels of candidate genes required for either pathway was detected. This study demonstrates that, in the seminiferous tubules, FSH suppression induces spermatogonial apoptosis predominantly via the intrinsic pathway, while spermatocyte apoptosis occurs via both the intrinsic and extrinsic pathways.

Journal of Endocrinology (2008) 197, 35-43

\section{Introduction}

Sperm output in adulthood relies on the establishment of the Sertoli cell population and initiation of germ cell development during foetal and early postnatal life. In rats, Sertoli cells proliferate from the time of gender specification at embryonic day 11.5 until around 15 days after birth (15 dpp), and this complement of Sertoli cells is sustained throughout adulthood (Orth et al. 1988). The first wave of spermatogenesis is initiated when gonocytes differentiate first into spermatogonia at $3 \mathrm{dpp}$; these first become spermatocytes at $\sim 9 \mathrm{dpp}$, form haploid spermatids by $18 \mathrm{dpp}$ and then first form spermatozoa at $43 \mathrm{dpp}$ (deRooij 1998).

Both Sertoli and germ cell populations are regulated during the first wave of spermatogenesis by endocrine signals including follicle-stimulating hormone (FSH) and luteinizing hormone (LH)/testosterone (T). A key role for FSH in regulating the size of the Sertoli cell population in early postnatal life has been attributed to its stimulatory effect on
Sertoli cell division (Orth 1984, Boitani et al. 1995, Orth et al. 1998, Meachem et al. 2005), with no evidence for an effect on survival (Meachem et al. 2005). We have previously demonstrated the absence of significant changes in apoptosis and proliferation rates of germ cells in $3 \mathrm{dpp}$ and 9 dpp rats following FSH suppression by immunoneutralization of the circulating hormone (Meachem et al. 2005). However, at 18 dpp, we documented a $2 \cdot 5$-fold increase in spermatogonial apoptosis (control versus FSH-suppressed groups: $6 \cdot 2 \pm 1 \cdot 0 \%$ vs $15 \cdot 5 \pm 1 \cdot 6 \% ; P<0 \cdot 001)$, suggesting that FSH acts as a germ cell survival factor rather than as a proliferative factor (Meachem et al. 2005). The apoptotic pathway(s) by which this germ cell apoptosis is (are) executed in response to FSH suppression is unknown.

Two pathways of apoptosis are described as active in the testis: the intrinsic and extrinsic pathways (reviewed in Sinha-Hikim et al. 2003a). The intrinsic pathway (or mitochondrial pathway) involves translocation of BAX from the cytosol to the mitochondria where it causes cytochrome $\mathrm{C}$ release into 
the cytosol. Cytochrome $\mathrm{C}$ then binds to the apoptotic protease activating factor-1, resulting in the activation of the initiator caspase 9 and subsequent activation of executioner caspases 3,6 and 7, leading to apoptosis (Adams \& Cory 1998, Green 2000, Hengartner 2000). The BCL2 protein family members, such as BCL2L2 (formerly BCL-W), have been shown to be involved in this pathway by the formation of dimers with BAX (Adams \& Cory 1998). The extrinsic pathway (or death receptor pathway) involves Fas ligand stimulation of FAS protein on target cells, which then activates the initiator caspase 8 , and subsequently activates executioner caspases, effecting apoptosis (Nagata \& Golstein 1995, Lee et al. 1997).

In adult rats, the intrinsic pathway has recently been shown to be involved in spermatogonial apoptosis following selective FSH suppression (Ruwanpura et al. 2007), while both the intrinsic and extrinsic pathways are involved in spermatocyte and spermatid apoptosis following selective $\mathrm{T}$ withdrawal (Nandi et al. 1999, Woolveridge et al. 1999). During the first wave of spermatogenesis, the extrinsic pathway of apoptosis functions during programmed germ cell apoptosis (Moreno et al. 2006, Codelia et al. 2007, Lizama et al. 2007). In immature (8-13 dpp) hpg mice lacking gonadotropins, spermatogonial and spermatocyte apoptosis occurs via both apoptotic pathways (Chausiaux et al. 2008). However, in immature rats, the regulation of each of these apoptotic pathways by hormones (FSH and/or LH/T) remains unknown.

To understand the mechanisms of FSH action on germ cell apoptosis in the immature rat in vivo, we have used the experimental model of FSH suppression by passive immunoneutralization with an anti-FSH antibody administered for 4 days to $14 \mathrm{dpp}$ normal rats (Meachem et al. 2005). We hypothesized that acute FSH suppression would result in accelerated germ cell apoptosis via activation of the intrinsic and/or extrinsic pathways. In this study, we aimed to determine the pathway(s) involved in germ cell apoptosis by employing antibody detection systems directed to the activated caspase forms (aCaspase 9: intrinsic pathway, aCaspase 8: extrinsic pathway) in combination with germ cell enumeration by stereology. In addition, we quantified the expression levels of candidate genes from both apoptotic pathways.

\section{Materials and Methods}

\section{Animals}

Testicular tissues were obtained from male 18 dpp outbred Sprague-Dawley rats after 4 days of acute FSH suppression starting on $14 \mathrm{dpp}$ (Meachem et al. 2005). All investigations conformed to the NHMRC/CSIRO/AAC Code of Practice for the Care and Use of Animals for Experimental Purposes and were approved by the Monash University Standing Committee on Ethics in Animal Experimentation.

\section{Experimental design}

Passive immunization against FSH Rats at $14 \mathrm{dpp}$ $(n=5$ per group) were immunized either with a purified in-house polyclonal ovine immunoglobulin fraction derived from antisera raised against rat FSH (FSHAb) or with normal sheep immunoglobulin (ConAb; Meachem et al. 1998, 2005). Each animal received a daily dose of FSHAb $(10 \mathrm{mg} / \mathrm{kg}$ in saline) by s.c. injections for 4 days, a fivefold higher dose of FSHAb (Meachem et al. 2005) capable of neutralizing $>90 \%$ of serum FSH levels in adult rats within $24 \mathrm{~h}$ (Meachem et al. 1998). FSHAb treatment does not alter the serum testosterone in this immature rat model (Meachem et al. 2005). Rats were killed $24 \mathrm{~h}$ after their final injection at $18 \mathrm{dpp}$.

Positive control tissues The heat-treated adult rat testis (courtesy of Dr Amiya Sinha Hikim) was used as the positive control for immunohistochemical identification of intrinsic pathway activation (Sinha-Hikim et al. 2003b).

\section{Tissue collection and preparation}

Testes were excised and the right testis of each rat snap frozen in liquid nitrogen and stored at $-80^{\circ} \mathrm{C}$ for total RNA preparation. The left testes were immersion fixed with Bouin's solution for $5 \mathrm{~h}$, weighed and then sampled (Meachem et al. 2005). The sampled tissues were then embedded in paraffin for immunohistochemistry. Prior to immunostaining, $5 \mu \mathrm{m}$ tissue sections were prepared, deparaffinized and hydrated through a series of progressively decreasing ethanol concentrations and rinsed with PBS (10 mM, pH 7•4).

\section{Assessment of apoptotic pathways}

The activation of apoptotic pathways has been assessed with a previously validated immunohistochemistry procedure employing antibodies against the activated forms of pathwayspecific caspases (Ruwanpura et al. 2007). In brief, aCaspase 9 antibody $(0.76 \mu \mathrm{g} / \mathrm{ml}$ in PBS; this rabbit polyclonal antibody detects p17 and p37 of aCaspase protein; Cell Signaling Technology, Danvers, MA, USA) was used to identify the intrinsic pathway activation, and aCaspase 8 antibody $(2.4 \mu \mathrm{g} / \mathrm{ml}$ in PBS; this mouse monoclonal antibody detects only the N-terminal region of the p18 subunit; Novocastra Laboratories, Newcastle, UK) was used for the extrinsic pathway detection. On negative control sections, the primary aCaspase 9 and 8 antibodies were substituted with the same concentration of rabbit and mouse IgG antibodies (Biosciences, Franklin Lakes, NJ, USA) respectively.

\section{Quantification of labelled cells}

Stereological techniques were applied to determine the percentages of aCaspase 9- or 8-labelled cells as described previously (Ruwanpura et al. 2007, 2008). Upon activation, 
caspases translocate from the cytoplasm to the nucleus; therefore intracellular localization of activated caspase varies during apoptotic pathway activation. The aCaspase-labelled cells were identified by brown nuclear, cytoplasmic and whole cell staining. Germ cell types were identified by their location within the seminiferous tubules, in conjunction with their nuclear size and shape. Cells within the seminiferous epithelium were classified as spermatogonia, spermatocytes or Sertoli cells (Russell et al. 1990). The percentages of labelled cells was assessed using an unbiased counting frame of $405 \mu \mathrm{m}^{2}$ per field superimposed on a video image by CASTGRID V1.60 software package (Olympus, Denmark, Germany), wherein 50-200 cell nuclei for each cell group were counted per rat. All slides were masked prior to the analysis. The extent of caspase activation was calculated by dividing the number of labelled cells by the total number of labelled and unlabelled cells in each group.

\section{Immunofluorescence and confocal studies}

To determine the prevalence of cells exhibiting the marker of each apoptotic pathway, the co-localization of TUNELlabelled cells with aCaspase 9 or 8 proteins was detected by confocal microscopy using immunofluorescent dual labelling (Ruwanpura et al. 2007, 2008). In situ detection of cells with DNA fragmentation was performed on tissue sections using an Apoptag fluorescein in situ apoptosis detection kit (Chemicon International, Temecula, MA, USA). For staining of aCaspases, antibodies to aCaspase 9 (Cell Signaling Technology) or aCaspase $8(2.4 \mu \mathrm{g} / \mathrm{ml}$ in PBS; this rabbit monoclonal antibody detects only the cleaved product $\mathrm{p} 18$, 41, 43 of active caspase 8 protein; Cell Signaling Technology) with secondary antibody goat anti-rabbit Alexa 546 secondary antibody (Molecular Probes, Eugene, OR, USA) were used. For negative control sections, TdT was omitted and the lack of secondary antibody cross-reactivity was verified by the substitution of the equivalent concentration of an isotype control antibody.

The proportions of TUNEL-labelled germ cells that were either aCaspase 9- or 8-positive were quantified by counting all the labelled and dual labelled cells, as described previously (Ruwanpura et al. 2007, 2008). TUNEL-labelled cells were observed with varying aCaspase staining intensity and were therefore designated as aCaspase-low or aCaspase-high. Only aCaspase-high TUNEL cells were included in the quantification for dual labelling.

\section{Total RNA extraction and reverse transcription (RT)}

Real-time PCR was used to measure the relative levels of five candidate apoptotic pathway-specific genes. Total RNA was extracted from testes treated with ConAb and FSHAb $(n=3$ per group), using a total RNA extraction kit (Qiagen). Any contaminating residual genomic DNA was removed using a DNAse-free kit (Ambion, Austin, TX, USA) according to the manufacturer's instructions. RNA (500 ng) was reverse transcribed to cDNA in a final volume of $20 \mu$ using Superscript III according to the manufacturer's protocol (Invitrogen). The absence of contaminating genomic DNA in total RNA samples was confirmed using reactions in which RT enzyme was omitted.

\section{Real-time PCR analysis}

Quantitative real-time PCR analysis was performed using the Roche LightCycler and the FastStart DNA Master SYBR Green I system (Roche). Oligonucleotide primer sequences specific for rat $\beta$-actin $(A c t b), B a x$ and $B c l 2 l 2$ were designed using Primer3 (www-genome.wi.mit.edu/cgi-bin/primer/ primer3_www.cgi), and Casp9 (caspase 9 gene), Casp 8 (caspase 8 gene) and Fas were obtained from published sources (Table 1). For PCR analyses, sample cDNA was diluted 1:4- to 1:80-fold. PCR conditions, including primer concentrations, $\mathrm{Mg}^{2+}$ concentration, annealing temperature and time, and extension time were optimized for each primer pair (Table 1). For all PCR analyses, standard curves were produced using dilutions of an immature $18 \mathrm{dpp}$ ConAb-treated rat testicular cDNA preparation assigned an arbitrary unitage. PCR of standards was performed using duplicate reactions, and samples were measured in triplicate for $\sim 38-40$ cycles, after which a melting curve analysis was performed to monitor PCR product purity (Table 1). Amplification of a single PCR product was confirmed by agarose gel electrophoresis and DNA sequencing (data not shown). To compare the expression levels of the apoptotic pathway-specific genes in groups, we normalized the data collected in our RNA analysis with the housekeeping gene, Actb.

\section{Statistical analysis}

All statistical analyses were performed using SigmaStat for Windows version 3.1 (Jandel Corporation, San Rafael, CA, USA). Data showing a normal distribution were analyzed using a $t$-test, and if data did not show normal distribution, Mann-Whitney test was performed. Data are expressed as mean \pm S.E.M. (for all histological data) or S.D. (for all gene expression levels), with $n=3-5$ rats per group as indicated.

\section{Results}

\section{FSH suppression increases intrinsic pathway activity}

In immature rats, 4 days of FSH suppression increased the proportion of TUNEL-labelled germ cells that were also positive for aCaspase 9 (by $1 \cdot 3$-fold) to $60 \cdot 5 \pm 2 \cdot 3 \%$ $(P=0 \cdot 03)$, compared with $53 \cdot 5 \pm 1 \cdot 6 \%$ in ConAb-treated samples (Fig. 1A), while no differences were seen in the proportion of aCaspase 8-positive, TUNEL-labelled germ cells between groups (Fig. 1C, D, F and G). 


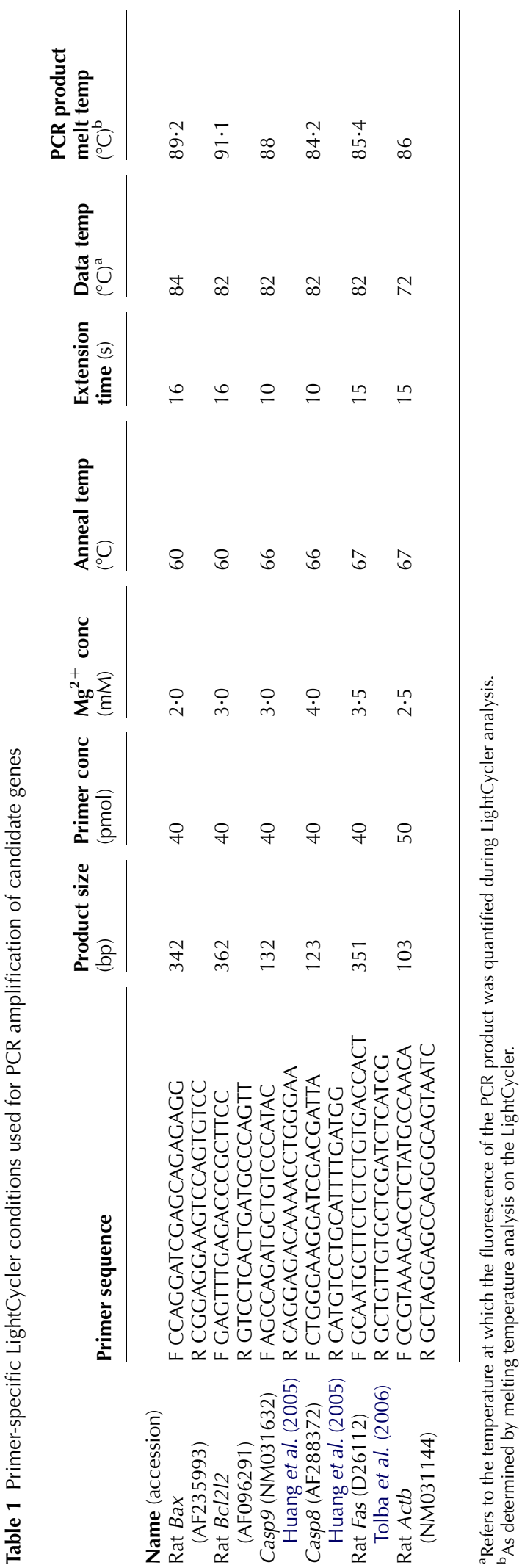

FSH suppression leads to germ cell apoptosis via the intrinsic apoptotic pathway

$\mathrm{FSHAb}$ treatment compared with ConAb treatment for 4 days resulted in a $9 \cdot 8$-fold increase in aCaspase 9-labelled spermatogonia $(0 \cdot 6 \pm 0 \cdot 4 \%$ vs $5 \cdot 6 \pm 0 \cdot 3 \% ; P<0 \cdot 001)$ and a $7 \cdot 5$-fold increase in aCaspase 9-labelled spermatocytes $(1 \cdot 6 \pm 0 \cdot 4 \%$ vs $11 \cdot 7 \pm 1 \cdot 1 \% ; P<0 \cdot 001 ;$ Fig. $2 \mathrm{~A}-\mathrm{C})$. No aCaspase 9-labelled Sertoli cells were observed in any samples (data not shown).

FSH suppression leads to spermatocyte apoptosis via the extrinsic apoptotic pathway

In immature rats treated with ConAb and FSHAb for 4 days, no aCaspase 8-labelled spermatogonia were observed. However, FSHAb treatment for 4 days resulted in a $5 \cdot 7$-fold increase in aCaspase 8-labelled spermatocytes $(2 \cdot 1 \pm 0 \cdot 8 \%$ vs $11 \cdot 8 \pm 1 \cdot 1 \% ; P<0 \cdot 001)$, compared with ConAb treatment (Fig. 3A-C). A few aCaspase 8-labelled Sertoli cells were observed in both ConAb- and FSHAbtreated rats (data not shown).

FSH suppression has no effect on intrinsic and extrinsic pathwayspecific candidate gene expression levels

Changes in the expression of three intrinsic pathway-specific genes, Casp9, Bcl2l2 and Bax, and two extrinsic pathwayspecific genes, Casp 8 and Fas, were examined by real-time PCR analysis of whole testis RNA. After 4 days of FSH suppression, consistent trends indicating reductions in Casp 9 (1·9-fold), Bcl2l2 (1·4-fold), Bax (1·4-fold), Casp8 (1·7-fold) and Fas $(1 \cdot 3$-fold) mRNA levels were seen compared with the ConAb treatment group; however, these did not achieve significance (Fig. 4).

\section{Discussion}

We demonstrate here that suppression of FSH affects the activity of both intrinsic and extrinsic apoptotic pathways in the immature rat testis in a cell-selective manner. We previously reported a $2 \cdot 5$-fold increase in spermatogonial apoptosis (control versus FSH-suppressed groups: $6 \cdot 2 \pm 1 \cdot 0 \%$ vs $15 \cdot 5 \pm 1 \cdot 6 \% ; \quad P<0 \cdot 001)$ occurs following acute $\mathrm{FSH}$ suppression for 4 days in $18 \mathrm{dpp}$ old rats (Meachem et al. 2005) and now show that this occurs principally via the intrinsic, rather than the extrinsic pathway. In addition, we show that FSH actions are important in spermatocyte survival through the regulation of both apoptotic pathways. No significant change in mRNA expression levels of pathwayspecific genes was documented. These data indicate that the mechanism by which FSH influences germ cell survival differs between germ cell subtypes during the first wave of spermatogenesis. 


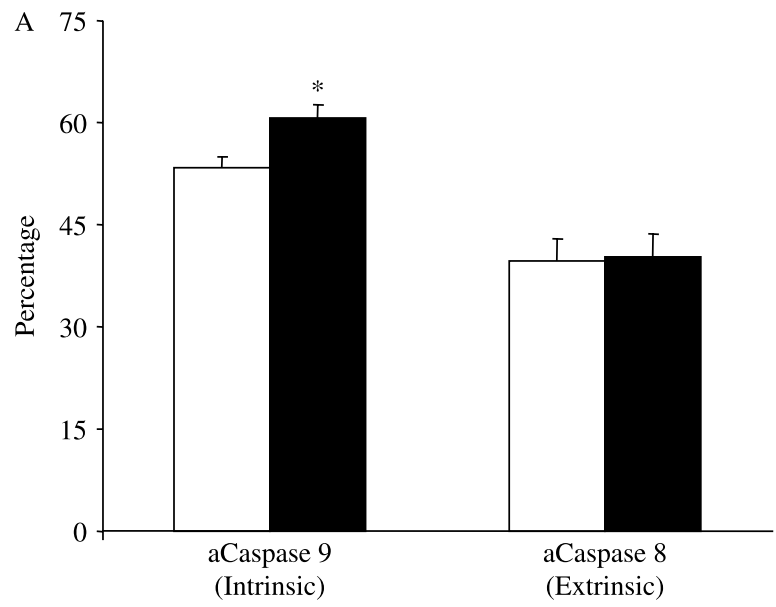

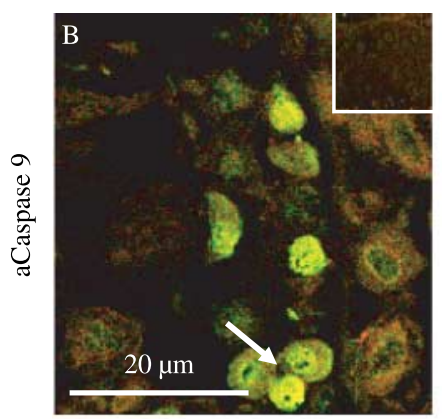

Heated QC

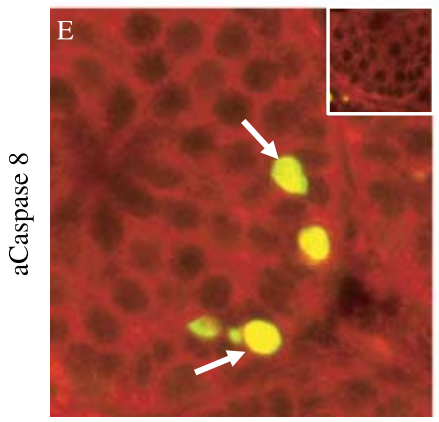

$\mathrm{ConAb}$

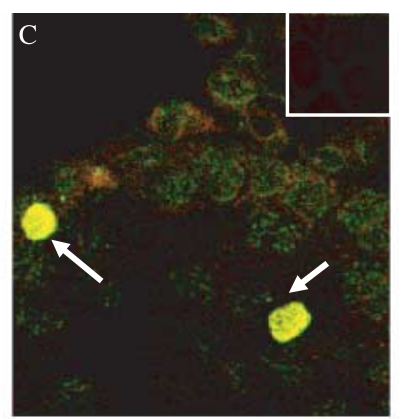

$\mathrm{ConAb}$

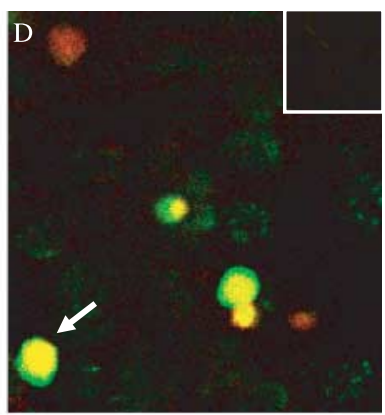

FSHAb

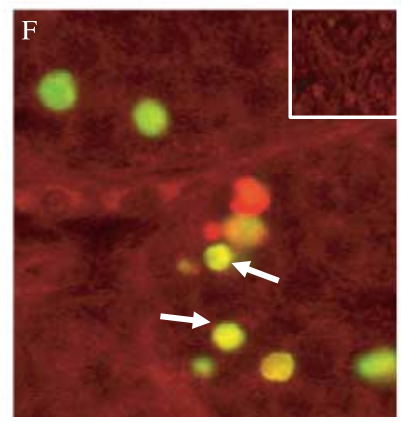

FSHAb

Figure 1 The proportion of TUNEL-labelled germ cells with aCaspase 9 or 8 reactivity (156/298 vs $130 / 213: 100 / 238$ vs 123/283 respectively) in $18 \mathrm{dpp}$ rats that had received ConAb (white bars) or FSHAb (black bars) for 4 days (A). Data are mean \pm S.E.M. ( $n=5$ per group). Asterisks denote significant differences between FSH-suppressed rats and the corresponding ConAb-treated rats $\left({ }^{*} P<0 \cdot 05\right)$. Photomicrographs of rat testis cross sections illustrate the presence of aCaspase $9(\mathrm{~B}-\mathrm{D})$ and $8(\mathrm{E}-\mathrm{F})$, both shown in red in rat germ cells with caspase activity. TUNEL-positive cells are shown in green. Dual labelling of caspases with TUNEL appears in yellow (see arrows). (B) shows a cross section of heated adult rat testis (intrinsic pathway quality control; see also Ruwanpura et al. 2007). (C) and (E) illustrate cells containing aCaspase 9 and 8 respectively in testes of rats that received ConAb for 4 days. (D) and (F) indicate aCaspase 9 and 8 respectively in testes of rats receiving FSHAb for 4 days. Inserts are negative controls incubated with an isotype-matched irrelevant antibody. 


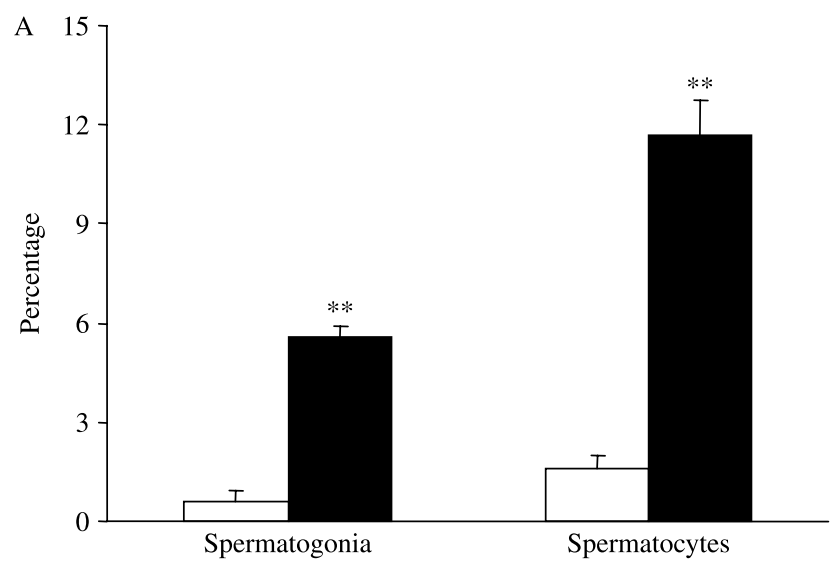

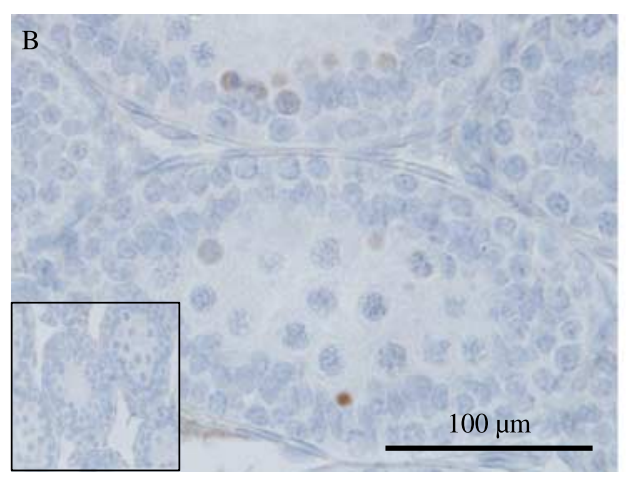

aCaspase 9 (intrinsic)

$\mathrm{ConAb}$

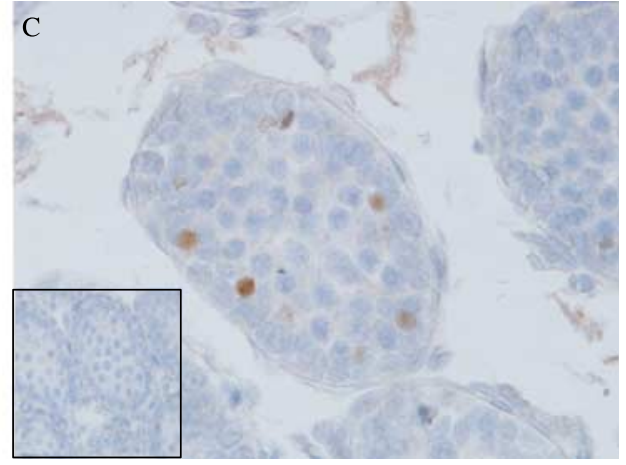

aCaspase 9 (intrinsic)

FSHAb

Figure 2 The percentage of aCaspase 9-labelled spermatogonia and spermatocytes (A), in 18 dpp rats that received ConAb (white bars) or FSHAb (black bars) for 4 days. Data are means \pm s.E.M. ( $n=5$ per group). Asterisks denote significant differences between FSH-suppressed rats in comparison with corresponding ConAb-treated samples $\left({ }^{* *} P<0 \cdot 001\right)$. Photomicrographs represent the staining of aCaspase 9 in testis cross sections for ConAb (B) and $\mathrm{FSHAb}(\mathrm{C})$ groups. Inserts illustrate the absence of a signal in negative controls.

This is the first demonstration that reduced FSH levels lead to decreased spermatogonial survival selectively via the intrinsic apoptotic pathway during the first wave of spermatogenesis. BAX, a component of the intrinsic pathway, is understood to function to control apoptosis in germ cells. In mice lacking Bax, spermatogonial numbers are elevated as the consequence of the failure of programmed apoptosis during the first wave of spermatogenesis (Knudson et al. 1995, Koji 2001). BAX protein also appears to be localized to spermatogonia at this time (Rodriguez et al. 1997). Similar to the findings presented here, we have previously shown that FSH withdrawal primarily affects intrinsic pathway activity in spermatogonia of adult rats (Ruwanpura et al. 2007). Data from gonadotropin-suppressed men demonstrated that gonadotropins regulate spermatogonial survival via only the intrinsic pathway, even though specific effects of FSH on spermatogonial survival on these men are unknown (Ruwanpura et al. 2008). However, the mechanism by which FSH regulates the intrinsic apoptotic pathway and spermatogonial development remains elusive. Based on knowledge from other systems, the actions of FSH-regulated factors could provoke changes in mitochondrial permeability, allowing factors such as cytochrome $C$ and BAX to be transported through the mitochondria, leading to apoptosis (Erkkila et al. 1999). The absence of various extrinsic pathway components in spermatogonia, such as FAS (Nandi et al. 1999), provides a simple explanation of why spermatogonial apoptosis via this pathway was not seen in mammals (D'Alessio et al. 2001, Chausiaux et al. 2008, Ruwanpura et al. 2007, 2008).

Our data suggest that FSH regulates both intrinsic and extrinsic apoptotic pathways during the meiotic phase of the first wave of spermatogenesis. This is in agreement with the observations of the phase when meiosis begins in immature (13 dpp) hpg mice. Chausiaux et al. (2008) reported increases in cleaved caspase 8 and 9 protein levels coinciding with increased spermatocyte apoptosis. During the first wave of spermatogenesis, physiological spermatocyte apoptosis correlates with BAX redistribution, cytochrome $\mathrm{C}$ redistribution and the activation of caspase 3 and FAS up-regulation (Lizama et al. 2007). 


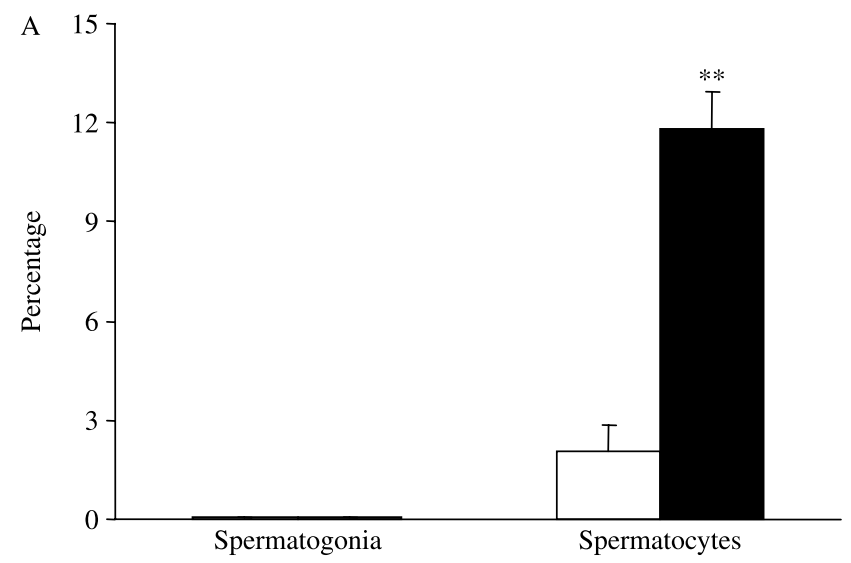

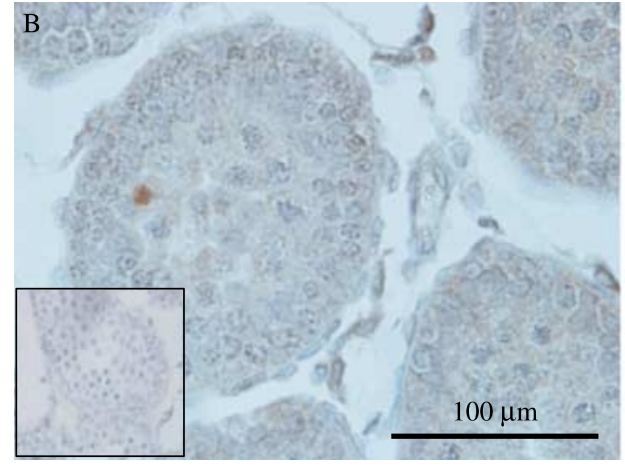

aCaspase 8 (extrinsic) $\mathrm{ConAb}$

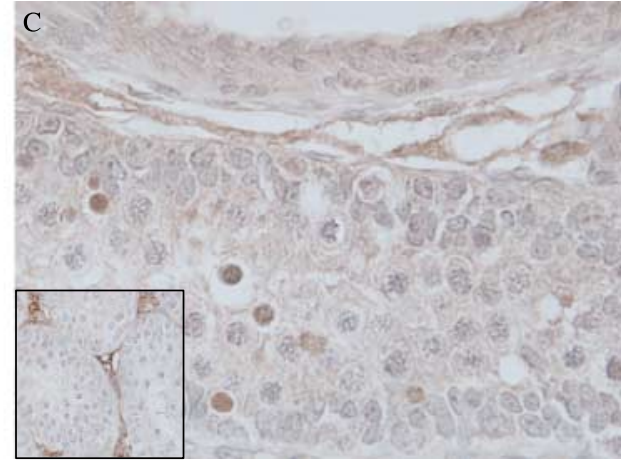

aCaspase 8 (extrinsic)

FSHAb

Figure 3 The percentage of aCaspase 8-labelled spermatogonia and spermatocytes (A) in ConAb (white bars) or FSHAb (black bars) samples. Data are means \pm s.E.M. $(n=5$ per group). Asterisks denote significant differences between treatment groups $(* * P<0 \cdot 001)$. Photomicrographs demonstrate aCaspase 8 signals in $\mathrm{ConAb}(\mathrm{B})$ and FSHAb (C) samples respectively. Inserts illustrate negative control signals.

Circulating FSH levels also affect spermatocyte and spermatid apoptosis via both pathways in FSH-suppressed adult rats and gonadotropin-suppressed men (Ruwanpura et al. 2007, 2008). It is of note that while previously we measured a $1 \cdot 5$-fold increase in spermatocyte apoptosis (control versus FSH-suppressed groups: $11 \cdot 8 \pm 1 \cdot 4 \%$ vs $16 \cdot 1 \pm 2 \cdot 2 \% ; P=0 \cdot 13)$ in FSHsuppressed immature rats (Meachem et al. 2005), we now report a six- to eightfold increase in caspase activity within this cell population. This difference most likely reflects the fact that initiator caspase activation is an upstream event in the apoptosis pathway, while TUNEL marks the latter phase of the pathway and such cells are rapidly lost.

Interestingly, in the subset of cells positive for TUNEL reactivity, FSH suppression only showed significant increases in the intrinsic pathways, but not in the extrinsic pathways (i.e. using dual labelling immunofluorescence). However, the significant increase in the proportion of aCaspase 8-labelled spermatocytes indicates that the approach employed was sufficient to detect extrinsic pathway activity in the germ cells (i.e. using single labelling immunohistochemistry). The apparent lack of change in caspase 8 activity recorded here for all apoptotic germ cells may be further refined using molecular markers to distinguish the subpopulations of these cells, and the samples in the present study will be suited for such an analysis when such markers that are compatible with the staining procedures used here become available. There may be another possible explanation for the no change in caspase 8 activity. It may be due to crosstalk between the apoptotic pathways. In many cell types, caspase 8 directly activates the executioner caspase, while in some cells FAS triggering induces the intrinsic pathway via a cleavage of the BCL2 protein family member, BID. BID can then induce BAX-mediated release of cytochrome $C$ from mitochondria, further committing the cell to apoptosis via the intrinsic pathway (Scaffidi et al. 1999, Said et al. 2004). This type of crosstalk has been seen for programmed spermatocyte apoptosis during the first wave of spermatogenesis in normal rats (Moreno et al. 2006, Lizama et al. 2007). However, we have not undertaken such analyses in this study. To our knowledge, there is no evidence for this crosstalk in other germ cell death models such as hormone deprivation, heat or genotoxic stress. 


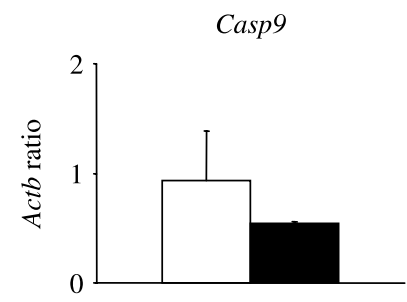

$B c l 2 l 2$
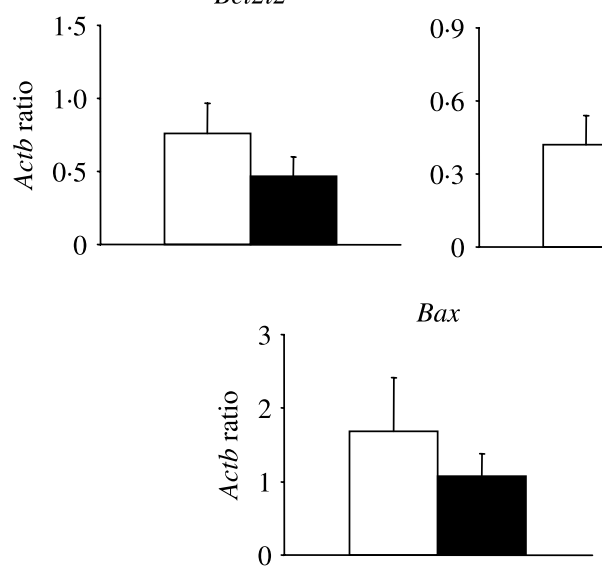

Figure 4 The mRNA levels of Casp9, BCl2/2, Bax, Casp8 and Fas in $\mathrm{ConAb}$ (white bars) or FSHAb (black bars) samples are presented following normalization to the Actb value for each sample. Data are mean \pm s.D. ( $n=3$ per group). No significant differences were recorded between treatment groups.

Interestingly, despite detection of both the intrinsic and extrinsic pathway-activated executioner proteins in germ cells, we observed no significant change in the transcript levels of Bax, Bcl2l2 and Fas following FSH suppression. However, the transcript levels may not necessarily reflect active protein concentration, due to the potential for post-translational regulation of protein activities. As active protein levels were not quantified in the present study, we cannot address these possibilities. In immature hpg mice, decreased Bax transcription was proposed to be due to androgen deficiency (Chausiaux et al. 2008), since Bax activity is androgen dependent in prostate cancer (Lin et al. 2006). Even though caspase activation is often thought of principally at the protein level controlling proteolytic cascades, some studies have reported increases in caspase $\mathrm{mRNA}$ due to apoptotic stimuli (Huang et al. 2005, Chausiaux et al. 2008). FSH suppression had no affect on the transcription of the initiator caspase genes in this study. On the other hand, in the hpg mouse testis, Casp 9 and Casp 8 expression levels were significantly increased, as assessed by microarray analysis (Chausiaux et al. 2008), although this was probably due to the deficiency in testosterone rather than lack of FSH.

In conclusion, this study reveals that FSH suppression regulates spermatogonial apoptosis via the intrinsic apoptotic pathway, while spermatocyte apoptosis occurs via both the intrinsic and extrinsic apoptotic pathways during the first wave of spermatogenesis. In this study, FSH has its effect at the protein levels for caspase 8 and caspase 9 , not at the transcript levels of various pathway-related genes. Understanding the basic mechanisms in which hormones regulate germ cell progression in the immature testis is an important step towards the understanding how normal testicular function is established.

\section{Acknowledgements}

We would like to thank Dr Amiya Sinha Hikim, HarborUniversity of California and Los Angeles Biomedical Research Institute, for providing heated testis tissues as the intrinsic pathway quality control, and Dr Pavel Sluka and $\mathrm{Mr}$ Simon Degen, Prince Henry's Institute, for designing PCR primers. Supported by the National Health and Medical Research Council of Australia, Program Grants (\#241000SJM, PGS \& RIM, \#334011-KLL), fellowship (\#384108KLL) and ARC COE (\#348239 KLL). The authors declare that there is no conflict of interest that would prejudice the impartiality of this scientific work.

\section{References}

Adams JM \& Cory S 1998 The Bcl-2 protein family: arbiters of cell survival. Science 281 1322-1326.

Boitani C, Stefanini M, Fragale A \& Morena AR 1995 Activin stimulates Sertoli cell proliferation in a defined period of rat testis development. Endocrinology 136 5438-5444.

Chausiaux OE, Abel MH, Baxter FO, Khaled WT, Ellis PJ, Charlton HM \& Affara NA 2008 Hypogonadal mouse, a model to study the effects of the endogenous lack of gonadotropins on apoptosis. Biology of Reproduction 78 77-90.

Codelia VA, Cisternas P \& Moreno RD 2007 Relevance of caspase activity during apoptosis in pubertal rat spermatogenesis. Molecular Reproduction and Development In press.

D'Alessio A, Riccioli A, Lauretti P, Padula F, Muciaccia B, De Cesaris P, Filippini A, Nagata S \& Ziparo E 2001 Testicular FasL is expressed by sperm cells. PNAS 98 3316-3321.

Erkkila K, Pentikainen V, Wikstrom M, Parvinen M \& Dunkel L 1999 Partial oxygen pressure and mitochondrial permeability transition affect germ cell apoptosis in the human testis. Journal of Clinical Endocrinology and Metabolism 84 4253-4259.

Green DR 2000 Apoptotic pathways: paper wraps stone blunts scissors. Cell $1021-4$.

Hengartner MO 2000 The biochemistry of apoptosis. Nature 407 770-776.

Huang W, Dobberfuhl A, Filippopoulos T, Ingelsson M, Fileta JB, Poulin AR \& Grosskreutz CL 2005 Transcriptional up regulation and activation of initiating caspases in experimental glaucoma. American Journal of Pathology $167673-681$.

Knudson CM, Tung KS, Tourtellotte WG, Brown GA \& Korsmeyer SJ 1995 Bax-deficient mice with lymphoid hyperplasia and male germ cell death. Science 270 96-99.

Koji T 2001 Male germ cell death in mouse testes: possible involvement of Fas and Fas ligand. Medical Electron Microscopy 34 213-222.

Lee J, Richburg JH, Younkin SC \& Boekelheide K 1997 The Fas system is a key regulator of germ cell apoptosis in the testis. Endocrinology 138 2081-2088.

Lin Y, Kokontis J, Tang F, Godfrey B, Liao S, Lin A, Chen Y \& Xiang J 2006 Androgen and its receptor promote Bax-mediated apoptosis. Molecular and Cellular Biology 26 1908-1916.

Lizama C, Alfaro I, Reyes JG \& Moreno RD 2007 Up-regulation of CD95 (Apo-1/Fas) is associated with spermatocyte apoptosis during the first round of spermatogenesis in the rat. Apoptosis 12 499-512. 
Meachem SJ, Wreford NG, Stanton PG, Robertson DM \& McLachlan RI 1998 Follicle-stimulating hormone is required for the initial phase of spermatogenic restoration in adult rats following gonadotropin suppression. Journal of Andrology 19 725-735.

Meachem SJ, Ruwanpura SM, Ziolkowski J, Ague JM, Skinner MK \& Loveland KL 2005 Developmentally distinct in vivo effects of FSH on proliferation and apoptosis during testis maturation. Journal of Endocrinology $186429-446$.

Moreno RD, Lizama C, Urzua N, Vergara SP \& Reyes JG 2006 Caspase activation throughout the first wave of spermatogenesis in the rat. Cell and Tissue Research 325 533-540.

Nagata S \& Golstein P 1995 The Fas death factor. Science 267 1449-1456.

Nandi S, Banerjee PP \& Zirkin BR 1999 Germ cell apoptosis in the testes of Sprague Dawley rats following testosterone withdrawal by ethane 1,2dimethanesulfonate administration: relationship to Fas? Biology of Reproduction 61 70-75.

Orth JM 1984 The role of follicle-stimulating hormone in controlling Sertoli cell proliferation in testes of fetal rats. Endocrinology 115 1248-1255.

Orth JM, Gunsalus GL \& Lamperti AA 1988 Evidence from Sertoli cell-depleted rats indicates that spermatid number in adults depends on numbers of Sertoli cells produced during perinatal development. Endocrinology 122 787-794.

Orth JM, McGuinness MP, Qiu J, Jester WF, Jr \& Li LH 1998 Use of in vitro systems to study male germ cell development in neonatal rats. Theriogenology 49 431-439.

Rodriguez I, Ody C, Araki K, Garcia I \& Vassalli P 1997 An early and massive wave of germinal cell apoptosis is required for the development of functional spermatogenesis. EMBO Journal 16 2262-2270.

de Rooij DG 1998 Stem cells in the testis. International Journal of Experimental Pathology 79 67-80.

Russell LD, Ettlin RA, Sinha-Hikim AP \& Clegg ED 1990 In Histological and Histopathological Evaluation of the Testis, Ed. LD Russell. Clearwater, Florida: Cache River Press.
Ruwanpura SM, McLachlan RI, Stanton PG \& Meachem SJ 2007 FSH affects spermatogonial survival by regulating the intrinsic pathway in adult rats. Biology of Reproduction In press.

Ruwanpura SM, McLachlan RI, Matthiesson KL \& Meachem SJ 2008 Gonadotropins regulate germ cell survival, not proliferation, in normal adult men. Human Reproduction 23 403-411.

Said TM, Paasch U, Glander HJ \& Agarwal A 2004 Role of caspases in male infertility. Human Reproduction Update 10 39-51.

Scaffidi C, Schmitz I, Zha J, Korsmeyer SJ, Krammer PH \& Peter ME 1999 Differential modulation of apoptosis sensitivity in CD95 type I and type II cells. Journal of Biological Chemistry 274 22532-22538.

Sinha-Hikim AP, Lue Y, Diaz-Romero M, Yen PH, Wang C \& Swerdloff RS $2003 a$ Deciphering the pathways of germ cell apoptosis in the testis. Journal of Steroid Biochemistry and Molecular Biology 85 175-182.

Sinha-Hikim AP, Lue Y, Yamamoto CM, Vera Y, Rodriguez S, Yen PH, Soeng K, Wang C \& Swerdloff RS $2003 b$ Key apoptotic pathways for heatinduced programmed germ cell death in the testis. Endocrinology 144 3167-3175.

Tolba RH, Schildberg FA, Schnurr C, Glatzel U, Decker D \& Minor T 2006 Reduced liver apoptosis after venous systemic oxygen persufflation in nonheart-beating donors. Journal of Investigative Surgery 19 219-227.

Woolveridge I, de Boer-Brouwer M, Taylor MF, Teerds KJ, Wu FC \& Morris ID 1999 Apoptosis in the rat spermatogenic epithelium following androgen withdrawal: changes in apoptosis-related genes. Biology of Reproduction $60461-470$.

Received in final form 22 January 2008

Accepted 29 January 2008

Made available online as an Accepted Preprint 29 January 2008 